\title{
A Study on the Effect of the Contact Point and the Contact Force of a Glass Fiber under End-Face Polishing Process
}

\author{
Ying-Chien Tsai, ${ }^{1}$ Guang-Miao Huang, ${ }^{2}$ Shin-Wei Cheng,, \\ Cheng-An Hsu, ${ }^{1}$ and Innchyn Her $^{2}$ \\ ${ }^{1}$ Department of Mechanical Engineering, Cheng Shiu University, Kaohsiung 833, Taiwan \\ ${ }^{2}$ Department of Mechanical \& Electro-Mechanical Engineering, National Sun Yat-Sen University, Kaohsiung 804, Taiwan
}

Correspondence should be addressed to Ying-Chien Tsai; yctsai@csu.edu.tw

Received 22 September 2014; Accepted 7 January 2015

Academic Editor: Aziz Dinia

Copyright (C) 2015 Ying-Chien Tsai et al. This is an open access article distributed under the Creative Commons Attribution License, which permits unrestricted use, distribution, and reproduction in any medium, provided the original work is properly cited.

\begin{abstract}
The offset between the center lines of the polished end-face and the fiber core has a significant effect on coupling efficiency. The initial contact point and the contact force are two of the most important parameters that induce the offset. This study proposes an image assistant method to find the initial contact point and a mathematical model to estimate the contact force when fabricating the double-variable-curvature end-face of single mode glass fiber. The repeatability of finding the initial contact point via the vision assistant program is $0.3 \mu \mathrm{m}$. Based on the assumption of a large deflection, a mathematical model is developed to study the relationship between the contact force and the displacement of the lapping film. In order to verify the feasibility of the mathematical model, experiments, as well as DEFORM simulations, are carried out. The results show that the contact forces are alomst linearly proportional to the feed amounts of the lapping film and the errors are less than $9 \%$. By using the method developed in this study, the offset between the grinding end-face and the center line of the fiber core is within 0.15 to $0.35 \mu \mathrm{m}$.
\end{abstract}

\section{Introduction}

A microlens improves the mode match between the laser source and single mode fiber by fabricating the tip of the single mode fiber as the microlens to allow for a direct coupling. The offset between the fiber center line and the microlens significantly affects the coupling efficiency. The coupling efficiency will drop $50 \%$ if the offset is more than $0.6 \mu \mathrm{m}$ on the horizontal axis [1]. There are three important factors to consider in reducing the offset of the asymmetric microlens. One is the quality of the broken edge of the optical fiber [2], the second is the initial contact point between the optical fiber and the lapping film [3], and the third is the control of the contact force between the optical fiber and the lapping film $[3,4]$.

The broken edge of the optical fiber will affect the offset if there is an angle between the normal of the broken edge and the center line of the optical fiber. The offset occurs when the material removal rate changes at a different spinning angle while the optical fiber is spinning. Normally, the initial contact point between the optical fiber and the lapping film is manually judged, and when a high material removal rate occurs it causes an undercut, which leads to the offset. The control of the contact force between the optical fiber and the lapping film affects the offset and profile of the end-face of the optical fiber at the same time. To improve coupling efficiency, the lens radius of the curvature on the vertical axis should be near $3 \mu \mathrm{m}$, the horizontal axis should be more than $25 \mu \mathrm{m}$, and the lapping tilt angle should be $45^{\circ}$ [5].

In this study, a mathematical model via the vision assistant method is presented. The determination of the original contact point will avoid an undercut, and the control of the contact force will keep a better profile on the end-face of the optical fiber.

\section{Determination of the Initial Contact Point}

Normally, the initial contact point between the optical fiber and the lapping film is manually determined. The optical fiber is slowly moved down toward the lapping film, and the initial contact point is determined when the operator finds the optical fiber is touching the turning lapping film and 
some chips are observed. However, this method needs skillful operators and the repeatability is not easy to control.

Tseng et al. [3] developed an online force sensor to automatically control the wedge-shaped end-face of the optical fiber polishing process, as shown in Figure 1. The offset between the fiber cladding and the fiber core is less than $1.5 \mu \mathrm{m}$.

In the present study, the vision recognition method was used to help find the initial contact point between the optical fiber and the lapping film. From Figure 2, some features can be noted and described as follows.

(i) The edge of the lapping film is not easy to observe in the image.

(ii) The lapping film may not be perfectly horizontal.

(iii) A reflection image of the optical fiber is shown in the image.

Finding the initial contact point between the fiber and the lapping film requires finding the lapping film and the end point of the edge of the optical fiber. Once this is done, the initial contact point and the distance between the optical fiber and the lapping film can be determined as shown in Figure 2.

2.1. Finding the Equations of the Edges of the Optical Fiber and Reflection Image. As shown in Figure 3, in drawing a line from the background to the optical fiber, there is a grey level intensity drop between the optical fiber and the background. By drawing several lines, the coordinates of the edge points are found and the least squares method is used to calculate the equation of the edge. In this way, all four edges of the optical fiber and the reflection image are found, as shown in Figure 3. The equations for all four edges are shown in (1) to (4), respectively:

$$
L_{i}: a_{i} x+b_{i} y+c_{i}=0,
$$

where $i=1,2,3$, and 4 .

2.2. Finding the Lapping Film. There are two methods for finding the line of the lapping film: the two-point method and the angular bisector method. Both methods are described below.

2.2.1. Two-Point Method. From Figure 3, lines $L_{1}$ and $L_{3}$ intersect at point $P_{1}$. The coordinates of point $P_{1}$ are shown in

$$
P_{1}\left(x_{p_{1}}, y_{p_{1}}\right)=\left(\frac{b_{1} c_{2}-b_{2} c_{1}}{a_{1} b_{2}-a_{2} b_{1}}, \frac{a_{1} c_{2}-a_{2} c_{1}}{a_{2} b_{1}-a_{1} b_{2}}\right) .
$$

Lines $L_{2}$ and $L_{4}$ intersect at point $P_{2}$. The coordinates of point $P_{2}$ are shown in

$$
P_{2}\left(x_{p_{2}}, y_{p_{2}}\right)=\left(\frac{b_{2} c_{4}-b_{4} c_{2}}{a_{2} b_{4}-a_{4} b_{2}}, \frac{a_{2} c_{4}-a_{4} c_{2}}{a_{4} b_{2}-a_{2} b_{4}}\right) .
$$

From (2) and (3), the lapping film equation can be derived, as shown in

$$
y-y_{p_{1}}=\frac{y_{p_{2}}-y_{p_{1}}}{x_{p_{2}}-x_{p_{1}}}\left(x-x_{p_{1}}\right) .
$$

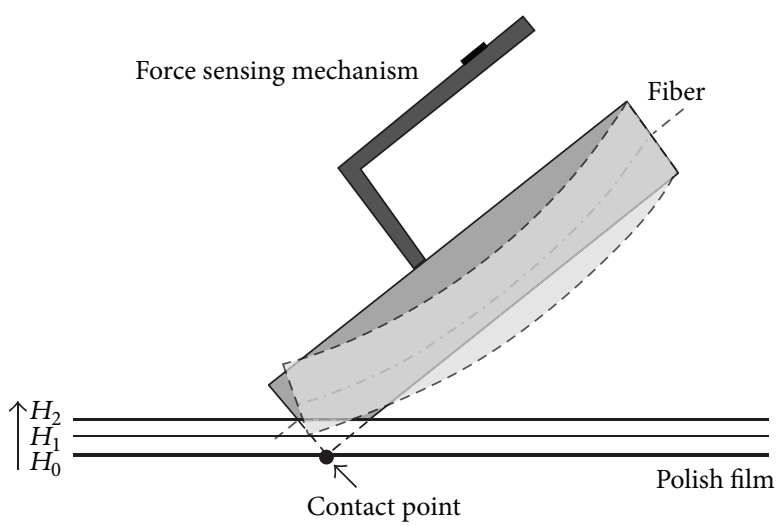

Figure 1: The force sensing mechanism [3].

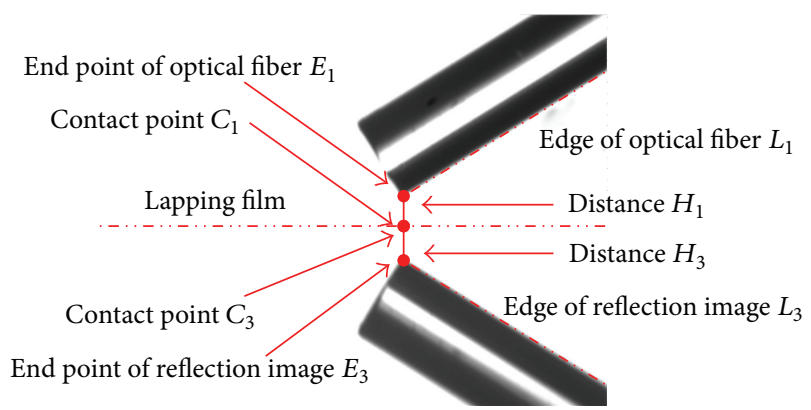

Figure 2: Photo of optical fiber and image of optical fiber.

2.2.2. Angular Bisector Method. Since the lapping film plays the role of a mirror, the slope of the lapping film should be equal to half of the sum of the slopes of lines $L_{1}$ and $L_{3}$, as shown in

$$
m_{\mathrm{lf}}=\frac{-a_{1} b_{2}-a_{2} b_{1}}{2 b_{1} b_{2}} .
$$

From (2) and (5), the lapping film equation can be derived, as shown in

$$
y-y_{p_{1}}=m_{\mathrm{lf}}\left(x-x_{p_{1}}\right) .
$$

Once the location of the lapping film is found, it is important to note if the lapping film is horizontal enough. If the lapping film is not perpendicular to the vertical displacement axis, $H$ axis, the contact force might be different from that expected and so affect the material removal rate.

2.3. Find the End Point of the Edges of the Optical Fiber and Reflection Image. A line drawn through the edge of optical fiber $L_{1}$ to intersection point $P_{1}$ shows there is a grey level intensity drop between the optical fiber and the background. The coordinates of the end point of the edge of the optical fiber, $E_{1}\left(x_{e_{1}}, y_{e_{1}}\right)$, are found, as shown in Figure 2. In the same way, the coordinates of the end point of the edge of the reflection image, $E_{3}\left(x_{e_{3}}, y_{e_{3}}\right)$, are also found.

2.4. Find the Contact Point and Distance. The coordinates of the edge points, $E_{1}\left(x_{e_{1}}, y_{e_{1}}\right)$ and $E_{3}\left(x_{e_{3}}, y_{e_{3}}\right)$, and the equation 


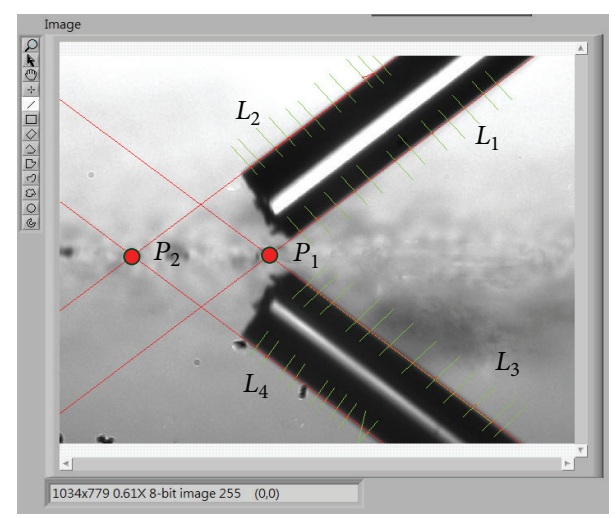

FIgURE 3: Edges of optical fiber and reflection image.

of the lapping film, $a_{\mathrm{lf}} x+b_{\mathrm{lf}} y+c_{\mathrm{lf}}=0$, are found. The contact points and distances are determined as follows:

$$
\begin{aligned}
& C_{1}\left(x_{c_{1}}, y_{c_{1}}\right)=\left[\begin{array}{l}
x_{c_{1}} \\
y_{c_{1}}
\end{array}\right]=\left[\begin{array}{c}
\frac{b_{\mathrm{lf}}^{2} x_{e_{1}}-a_{\mathrm{lf}} b_{\mathrm{lf}} y_{e_{1}}-a_{\mathrm{lf}} c_{\mathrm{lf}}}{\sqrt{a_{\mathrm{ff}}^{2}+b_{\mathrm{lf}}^{2}}} \\
\frac{a_{\mathrm{lf}}^{2} y_{e_{1}}-a_{\mathrm{lf}} b_{\mathrm{lf}} x_{e_{1}}-b_{\mathrm{lf}} \mathfrak{c}_{\mathrm{lf}}}{\sqrt{a_{\mathrm{ff}}^{2}+b_{\mathrm{lf}}^{2}}}
\end{array}\right] \text {, } \\
& H_{1}=\frac{\left|a_{\mathrm{lf}} x_{e_{1}}+b_{\mathrm{lf}} y_{e_{1}}+c_{\mathrm{lf}}\right|}{\sqrt{a_{\mathrm{lf}}^{2}+b_{\mathrm{lf}}^{2}}}, \\
& C_{3}\left(x_{c_{3}}, y_{c_{3}}\right)=\left[\begin{array}{c}
x_{c_{3}} \\
y_{c_{3}}
\end{array}\right]=\left[\begin{array}{c}
\frac{b_{\mathrm{ff}}^{2} x_{e_{3}}-a_{\mathrm{lf}} b_{\mathrm{lf}} y_{e_{3}}-a_{\mathrm{lf}} c_{\mathrm{lf}}}{\sqrt{a_{\mathrm{lf}}^{2}+b_{\mathrm{lf}}^{2}}} \\
\frac{a_{\mathrm{lf}}^{2} y_{e_{3}}-a_{\mathrm{lf}} b_{\mathrm{lf}} x_{e_{3}}-b_{\mathrm{lf}} c_{\mathrm{lf}}}{\sqrt{a_{\mathrm{lf}}^{2}+b_{\mathrm{lf}}^{2}}}
\end{array}\right] \text {, } \\
& H_{1}=\frac{\left|a_{\mathrm{lf}} x_{e_{3}}+b_{\mathrm{lf}} y_{e_{3}}+c_{\mathrm{lf}}\right|}{\sqrt{a_{\mathrm{lf}}^{2}+b_{\mathrm{lf}}^{2}}} \text {. }
\end{aligned}
$$

\section{Mathematical Model of the Contact Force}

The polishing mechanism of the glass was widely discussed $[4,6,7]$. In this study, the Preston equation [4] was used as the governing equation for the polishing operation. The material removal rate (MRR) can be expressed as follows:

$$
\frac{d H}{d t}=R_{H}=K \frac{N}{A} V
$$

where $K$ is the Preston coefficient $\left(\mu \mathrm{m}^{2} / \mathrm{mN}\right), R_{H}$ is the thickness removal rate $(\mu \mathrm{m} / \mathrm{s}), N$ is the contact force $(\mathrm{mN})$, $A$ is the polishing area $\left(\mu \mathrm{m}^{2}\right)$, and $V$ is the polishing velocity $(\mu \mathrm{m} / \mathrm{s})$.

Since the Preston equation is derived from plate glass polishing, (8) should be modified if the polishing area varies with time.

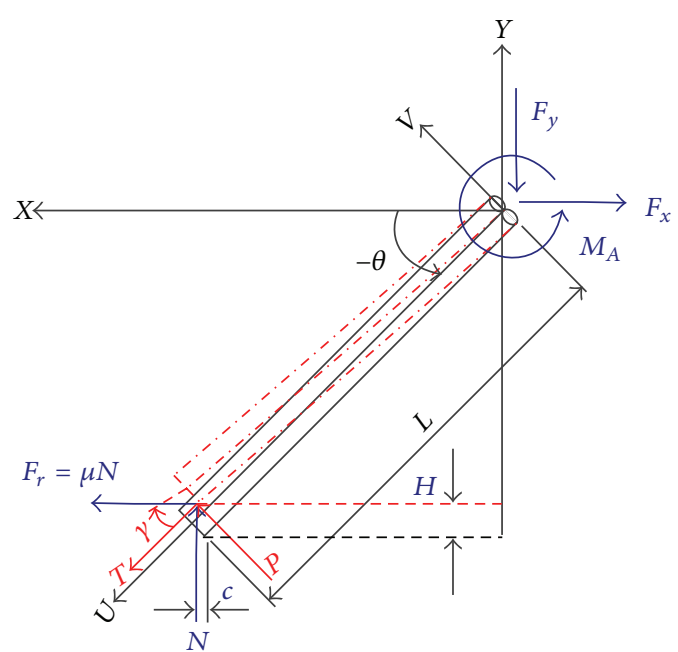

FIGURE 4: Free body diagram of the polishing process.

Since $d Q=A d H$, the MRR is modified as follows:

$$
\frac{d Q}{d t}=R_{Q}=K N V
$$

where $R_{\mathrm{Q}}$ is the volume removal rate $\left(\mu \mathrm{m}^{3} / \mathrm{s}\right)$.

From (9), the volume removal rate $R_{Q}$ is proportional to polishing velocity $V$ and contact force $N$. The polishing velocity is determined by setting the speed of the abrasive pad and work piece and is held constant during the polishing operation. The contact force is proportional to the displacement of the contact point. Once the displacement of the contact point is known, the contact force can be derived. The free body diagram of the optical fiber is shown in Figure 4 . Deflection is defined as positive if it is upward and the deflection angle is defined as positive if it is clockwise. The body weight of the optical fiber is neglected since the effect is quite small compared with the contact force.

3.1. Coordinate Transformation between XY and UV Coordinate Systems. As Figure 4 shows, the lapping film moves a distance, $H$, upward in the $X Y$ coordinate system. The new positions of the contact points in the $X Y$ and $U V$ coordinate systems are shown in (10) and (11), respectively:

$E_{1}\left(x_{e_{1}}, y_{e_{1}}\right)=(L \cos \theta-R \sin \theta+c,-L \sin \theta-R \cos \theta+H)$

$$
E_{1}\left(u_{e_{1}}, v_{e_{1}}\right)=\left(u_{0}+R \sin \gamma_{0}, v_{0}-R \cos \gamma_{0}\right)
$$

where $R$ is the radius of the optical fiber, $\theta$ is the tilt angle, $c$ is the horizontal displacement of the contact point in the $X Y$ coordinate system, $H$ is the vertical displacement of the contact point in the $X Y$ coordinate system, $u$ and $v$ are the coordinates of the end point of the neutral axis in the $U V$ coordinate system, and $\gamma_{0}$ is the inclined angle of the end point of the neutral axis in the $U V$ coordinate system. 


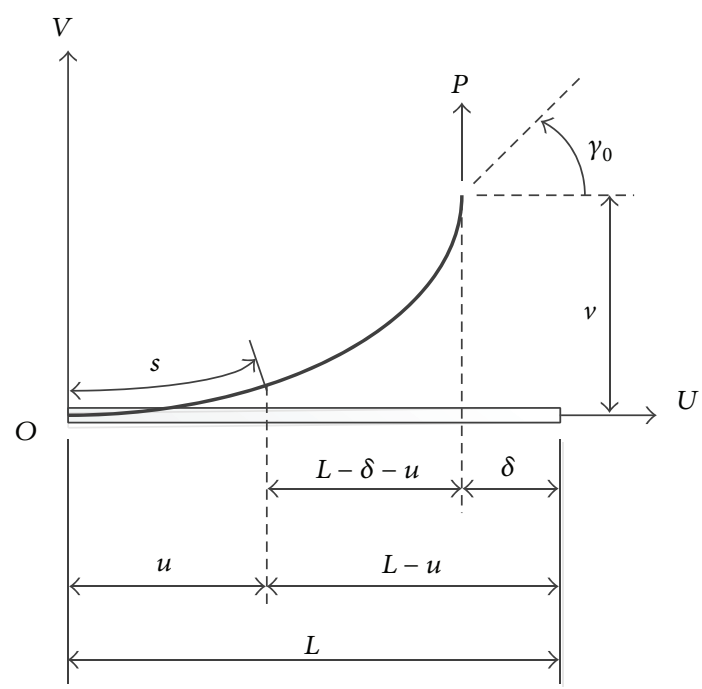

FIGURE 5: Large deflection of cantilever beam under concentrated load.

According to the coordinate transformation method, the relation of the contact point between the $X Y$ coordinates and $U V$ coordinates is

$\left[\begin{array}{c}u_{0}+R \sin \gamma_{0} \\ v_{0}-R \cos \gamma_{0}\end{array}\right]=\left[\begin{array}{cc}\cos \theta & -\sin \theta \\ \sin \theta & \cos \theta\end{array}\right]\left[\begin{array}{c}L \cos \theta-R \sin \theta+c \\ -L \sin \theta-R \cos \theta+H\end{array}\right]$.

From (12), the coordinate of the free end point of the neutral axis $\left(u_{0}, v_{0}\right)$ can be found. Then, the displacement of the free end point of the neutral axis can be calculated. The displacement of the free end point of the neutral axis is helpful in determining the contact force.

\subsection{Large Deflection of Cantilever Beam under Concentrated} Load. From the large deflection of cantilever beam theory [8], the relation between inclined angle $\gamma$ and concentrated load $P$ is shown in Figure 5:

$$
E I \frac{d^{2} \gamma}{d s^{2}}+P \cos \gamma=0
$$

Let

$$
\begin{aligned}
\alpha^{2} & =\frac{P L^{2}}{E I}, \\
2 k^{2} & =1+\sin \gamma_{0}, \\
\sin \tau_{1} & =\frac{\sqrt{2}}{2 k} .
\end{aligned}
$$

The deflection of the cantilever beam can be calculated as follows:

$$
\begin{aligned}
\alpha & =F(k)-F\left(k, \tau_{1}\right), \\
v_{0} & =L\left[1-\frac{2}{\alpha}\left(E(k)-E\left(k, \tau_{1}\right)\right)\right], \\
u_{0} & =L-\delta=L \frac{\sqrt{2 \sin \gamma_{0}}}{\alpha},
\end{aligned}
$$

where $E$ is Young's modulus; $I$ is the second moment of area of the beam's cross section; $\gamma_{0}$ is the inclined angle of the free end point of the neutral axis; $L$ is the length of the cantilever beam; $u_{0}$ is the coordinate of the free end point of the neutral axis in the $U$ axis; $v_{0}$ is the coordinate of the free end point of the neutral axis in the $V$ axis; and $F(k), F\left(k, \tau_{1}\right), E(k)$, and $E\left(k, \tau_{1}\right)$ are complete and incomplete elliptic integrals of the first and second kinds, respectively.

The displacement of the contact point in the XY coordinate system is observed from the vision image. The displacement of the free end point of the neutral axis in the $U V$ coordinate system can be derived by using the golden section algorithm to find the minimization of

$$
F\left(\gamma_{0}\right)=\left(u_{0}+R \sin \gamma_{0}-\alpha\right)^{2}+\left(v_{0}-R \cos \gamma_{0}-b\right)^{2},
$$

where

$$
\begin{aligned}
& 0 \leq \gamma_{0} \leq \frac{\pi}{2}, \\
& {\left[\begin{array}{l}
a \\
b
\end{array}\right]=\left[\begin{array}{cc}
\cos \theta & -\sin \theta \\
\sin \theta & \cos \theta
\end{array}\right]\left[\begin{array}{c}
L \cos \theta-R \sin \theta+c \\
-l \sin \theta-R \cos \theta+H
\end{array}\right] .}
\end{aligned}
$$

From (14) to (16), seven unknown variables, $\gamma_{0}, \alpha, k, \tau_{1}$, $v_{0}, u_{0}$, and $P$, will be solved. Since $\vec{P}+\vec{T}=\overrightarrow{F_{r}}+\vec{N}$, contact force $N$ can be derived from

$$
N=\frac{P}{(\cos \theta+\zeta \mu \sin \theta)}
$$

where $\zeta$ is equal to +1 or -1 depending on the direction of the polishing force, $F_{r}$.

\section{Experiment Results}

4.1. Original Contact Point. The third generation optical fiber polishing machine, developed by the authors, was used to evaluate the result of the original contact point, as shown in Figure 6. A vision assistant program was developed by using LabView software to find the distance between the optical fiber and the lapping film, as shown in Figure 7. The unit shown in Figure 7 is in pixels. The diameter of the optical fiber shown in Figure 7 is 164.5 pixels, corresponding to $125 \mu \mathrm{m}$, and the distance variation between $H_{1}$ and $H_{3}$ is 0.3 pixels, corresponding to $0.23 \mu \mathrm{m}$.

A repeatability comparison between the two-point method and the angular bisector method was made. Table 1 shows the average results of the 30 experiments for each method. The variation ratio is defined as the percentage of the standard deviation divided by the average. As shown in Table 1, all variation ratios of the angular bisector method were much better than those of the two-point method. The repeatability of the angular bisector method was also much better than that of the two-point method. The two-point method had a poorer performance as the image quality of the edge of the reflection image, $L_{4}$, was less clear.

An experiment was conducted for comparing the difference between manual judging and the vision assistant program. First, the lapping film was stuck on the turntable, 
TABLE 1: Repeatability between the two-point and angular bisector methods.

\begin{tabular}{lcccc}
\hline & & $\begin{array}{c}\text { Diameter of optical fiber } \\
\phi d \text { (pixel) }\end{array}$ & $\begin{array}{c}\text { Distance between optical } \\
\text { fiber and lapping film } \\
H_{1} \text { (pixel) }\end{array}$ & \multicolumn{2}{c}{$\begin{array}{c}\text { Distance between fiber } \\
\text { image and lapping film } \\
H_{3} \text { (pixel) }\end{array}$} & 29.78 \\
\hline \multirow{3}{*}{ Two-point method } & Average & 164.70 & 31.14 & 3.40 \\
& Standard deviation & 1.59 & 2.21 & $\mathbf{1 1 . 4 3 \%}$ \\
\hline \multirow{2}{*}{$\begin{array}{l}\text { Angular bisector } \\
\text { method }\end{array}$} & Variation ratio & $\mathbf{0 . 9 6 \%}$ & 23.06 & 23.06 \\
& Average & 156.01 & 0.39 & 0.39 \\
\hline
\end{tabular}

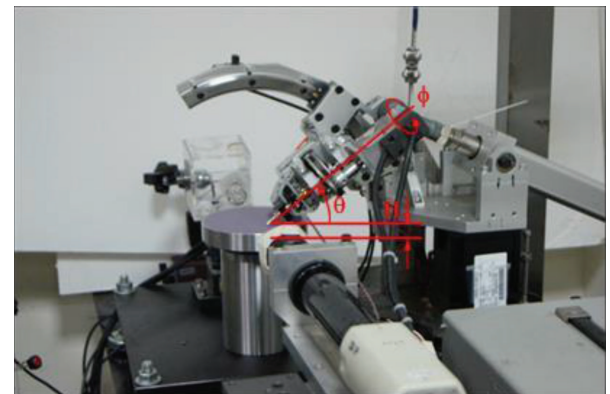

Figure 6: Photo of the 3rd generation optical fiber polishing machine.

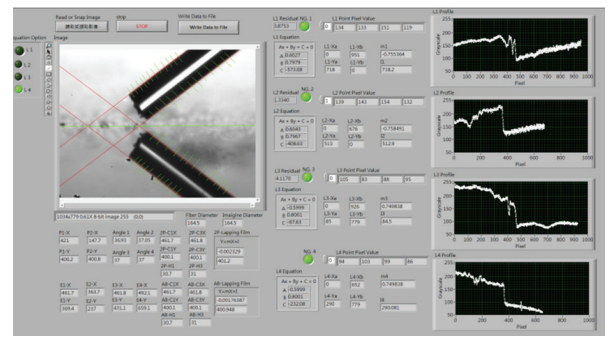

FIGURE 7: Photo of the front panel of the vision assistant program.

which was then turned. Then, an optical fiber with cladding and core only was prepared, and the prepared optical fiber was put into a spinning axis. Finally, the optical fiber was moved downward until it touched the turning lapping film, judged either manually or using the vision assistant program. As Figure 8 shows, manual judging resulted in a bigger undercut than that of using the vision assistant program.

4.2. Contact Force. Since the contact force was only up to several grams, a low-friction measuring stand was developed, as shown in Figure 9. A New Way air bushing was used to reduce the thrust load because the coefficient of friction of the air bushing was within 0.00001. An IMADA force gauge with accuracy up to 0.1 grams was used to measure the contact force.

In addition to the experiments, a DEFORM simulation and mathematical model were used to compare the results.
The parameters were set to fit the conditions of the optical fiber polishing process. The length of the optical fiber was $1800 \mu \mathrm{m}$, the cladding dimension of the optical fiber was $125 \mu \mathrm{m}$, Young's modulus of the single mode glass fiber was $73 \mathrm{GPa}$, and the tensile strength was $350 \mathrm{MPa}$. Three tilt angles, $30^{\circ}, 45^{\circ}$, and $60^{\circ}$, were set to compare with the results.

Solving (16) through the assistance of the vision image, the dimensionless positions of the contact point and neutral point were as shown in Figure 10. The scales of the $U$ axis and $V$ axis are different in order to show the characteristics of the contact point and the neutral point. The trajectory of the contact point is a straight line because the contact point only slides on the lapping film. The trajectory of the neutral point is a curve because the motion of the neutral point is the combination of translation and rotation.

The comparison of the experiment, simulation, and mathematical model for the $Y$ displacement of the contact point and the contact force is shown in Figure 11. The contact forces for the experiments were linear, except for the last two sets of data of the $60^{\circ}$ tilt angle. The reason for this was that the contact force became too large, which induced large friction force which prevented the movement of the optical fiber or even the breaking of the optical fiber. Thus, the last two sets of data of the $60^{\circ}$ tilt angle became nonlinear and then the fiber was broken. The data of the experiments were very close to the data of the DEFORM simulations. The contact force was proportional to the angle for the same $Y$ displacement of the contact point, which meant that the greater the tilt angle, the higher the material removal rate.

The errors of contact force $N$ between the mathematical models and experiments were $5.3 \%, 7.0 \%$, and $9.0 \%$ for tilt angles of $30^{\circ}, 45^{\circ}$, and $60^{\circ}$, respectively, as shown in Table 2 . The errors of the mathematical models were caused by the friction force. Normally there were three friction forces exerted on the contact point of the optical fiber, which would enlarge the contact force. In this study, the mathematical model simplified the friction force as one friction force in $X Y$ plane, which would underestimate the amount of contact force and cause the errors.

As shown in Figure 11, the contact force increased linearly at each tilt angle, $30^{\circ}, 45^{\circ}$, and $60^{\circ}$. The material removal rate was proportional to the contact force from (12) and proportional to the displacement of the $H$ axis. The ratio of MRR of the experiments at $30^{\circ}, 45^{\circ}$, and $60^{\circ}$ was close to 


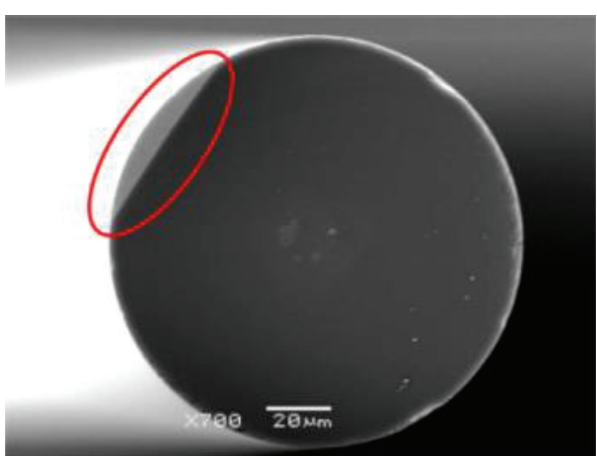

(a) Manual judging

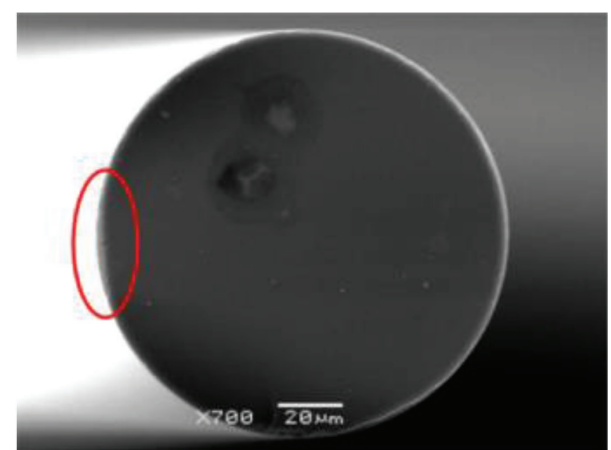

(b) Vision assistant program

FIGURE 8: SEM photos of the end-face of the optical fiber.

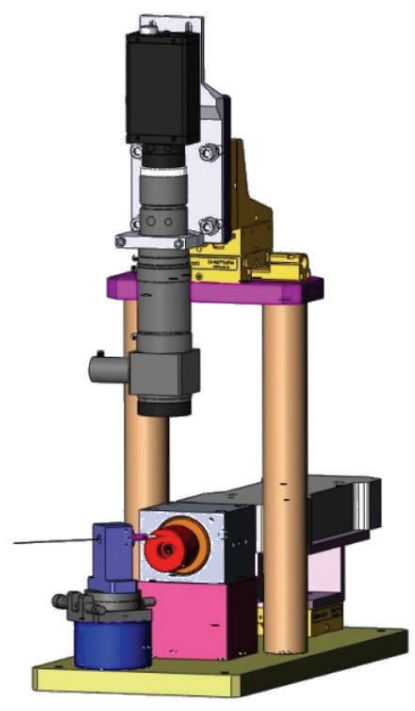

(a) CAD model

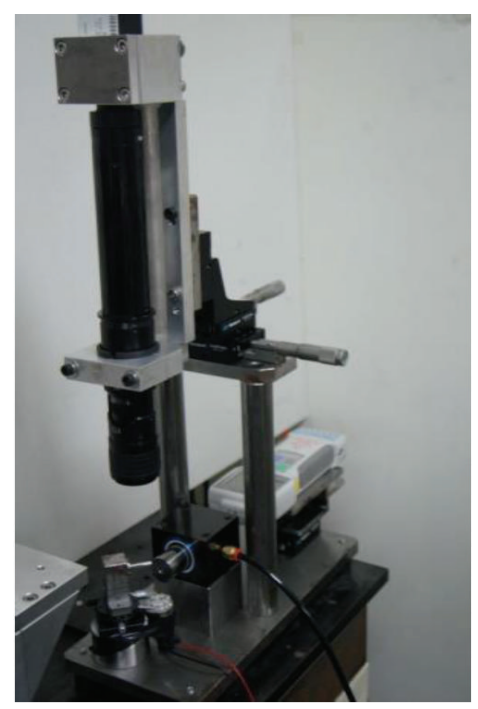

(b) Photo

FIgURE 9: Contact force measuring stand.

$4: 6: 13$, as shown in Table 2 . The profile of the end-face could be easily determined since the MRR was proportional to the displacement of the $H$ axis.

To improve coupling efficiency, the lens curvature radius in the major axis, Rlx, was set as $30 \mu \mathrm{m}$, the lens curvature radius in the minor axis, Rly, was set as $3 \mu \mathrm{m}$, and the lapping tilt angle was set as $45^{\circ}$. Using the vision assistant, the offset between the polishing end-face in the minor axis and the center line of the fiber core was controlled to within $0.4 \mu \mathrm{m}$, as shown in Figure 12.

\section{Conclusions}

In this study, the image assistant method was successfully applied to find the initial contact point and to estimate the contact force when fabricating the elliptical end-face of glass fiber. A vision assistant program was built to find the initial contact point to avoid the undercut and to reduce the offset between the fiber core and the end-face. Further, a mathematical model was developed to describe the relationship between the contact force and the displacement of the lapping film and to assist in determining the feed amount of the lapping film. The main results can be summarized as follows.

(i) The vision assistant program showed that the repeatability of the distance between the optical fiber and the lapping film was 0.39 pixels, which corresponds to $0.3 \mu \mathrm{m}$. The vision assistant program also helped verify whether the lapping film was horizontal enough to cause a negative effect. The contact experiments demonstrated that this program would avoid undercutting more effectively than manual judging.

(ii) According to the mathematical model, the relationship between the contact force and the displacement of the lapping film, $H$ axis, was linear. When compared with the results of the mathematical model, both experiments and DEFORM simulations showed the same tendency. Considering the mathematical model as the reference, the errors between the mathematical models and the experiments were 5.3\%, 
TABLE 2: Slopes and errors of contact force $N$ versus displacement $Y$.

\begin{tabular}{lcccc}
\hline Tilt angle (degree) & Slope (experiment) & Slope (DEFORM simulation) & Slope (mathematical model) & Errors (exp-math)/math \\
\hline 30 & 0.6511 & 0.6541 & 0.6181 & $5.3 \%$ \\
45 & 1.0134 & 1.035 & 0.9469 & $7.0 \%$ \\
60 & 2.1349 & 2.235 & 1.9586 & $9.0 \%$ \\
\hline
\end{tabular}

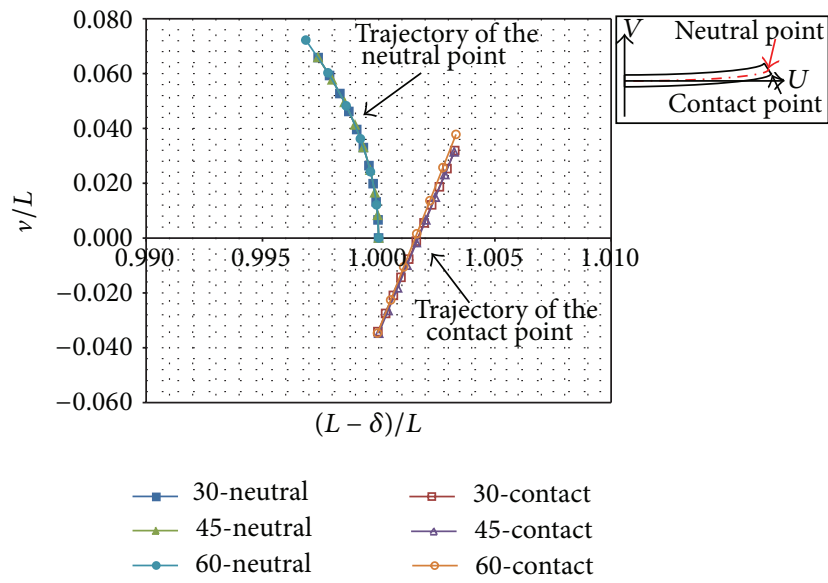

FIgure 10: Dimensionless positions of neutral point and contact point.

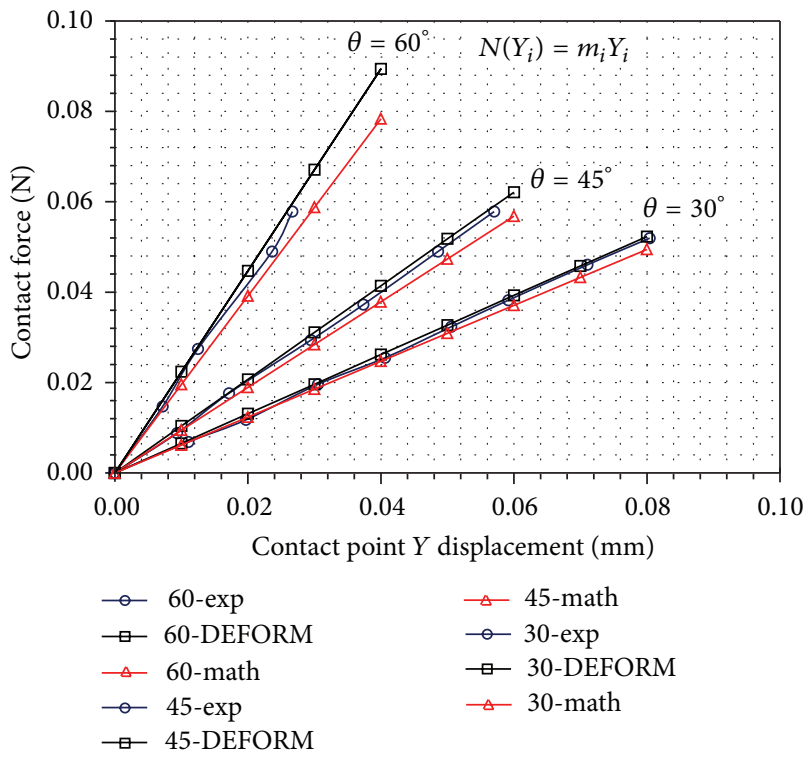

FIGURE 11: Comparison of simulation and mathematical model.

$7.0 \%$, and $9.0 \%$ for tilt angles of $30^{\circ}, 45^{\circ}$, and $60^{\circ}$, respectively.

(iii) The horizontal axis offsets between the fiber core and end-face were successfully controlled within 0.15 to $0.35 \mu \mathrm{m}$. The average offset of horizontal axis was $0.26 \mu \mathrm{m}$.

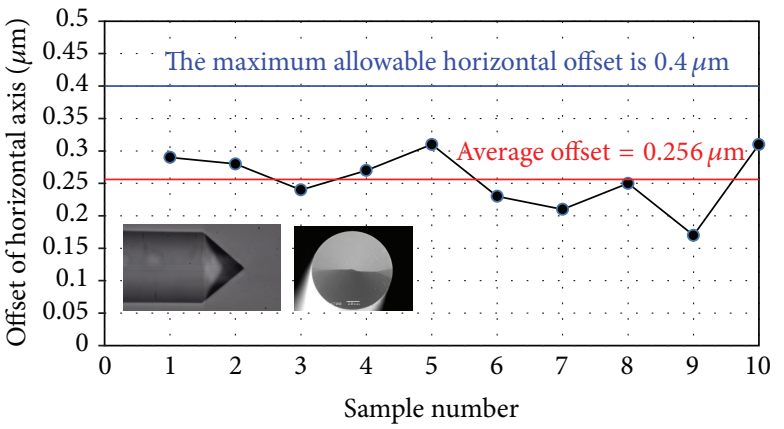

FIGURE 12: Offset between grinding end-face and center line of fiber core at $45^{\circ}$ tilt angle.

\section{Conflict of Interests}

The authors declare that there is no conflict of interests regarding the publication of this paper.

\section{Acknowledgment}

This work was supported by the National Science Council, Taiwan, under Contracts NSC 102-2221-E-230-005.

\section{References}

[1] R. A. Modavis and T. W. Webb, "Anamorphic microlens for laser diode to single-mode fiber coupling," IEEE Photonics Technology Letters, vol. 7, no. 7, pp. 798-800, 1995.

[2] Y.-C. Tsai, G.-M. Huang, J.-H. Chen, and S.-W. Chen, "The effect of the broken edge on the concentricity of the micro-polished optical fiber end-face," Applied Mechanics and Materials, vol. 284-287, pp. 2778-2783, 2013.

[3] Y.-T. Tseng, T.-Y. Hung, J.-H. Liu, and C.-H. Chang, "Optical fiber polishing automation with on-line force sensing," International Journal of Machine Tools and Manufacture, vol. 47, no. 6, pp. 892-899, 2007.

[4] F. W. Preston, "The theory and design of plate glass polishing machines," Journal of the Society of Glass Technology, vol. 11, pp. 214-256, 1927.

[5] Y.-D. Liu, Y.-C. Tsai, Y.-K. Lu et al., "New scheme of doublevariable-curvature microlens for efficient coupling high-power lasers to single-mode fibers," Journal of Lightwave Technology, vol. 29, no. 6, Article ID 5678609, pp. 898-904, 2011.

[6] C. J. Evans, E. Paul, D. Dornfield et al., "Material removal mechanisms in lapping and polishing," CIRP Annals, vol. 52, no. 2, pp. 611-633, 2003.

[7] N. Belkhir, D. Bouzid, and V. Herold, "Wear behavior of the abrasive grains used in optical glass polishing," Journal of 
Materials Processing Technology, vol. 209, no. 20, pp. 6140-6145, 2009.

[8] K. E. Bisshopp and D. C. Drucker, "Large deflection of cantilever beams," Quarterly of Applied Mathematics, vol. 3, pp. 272-275, 1945. 

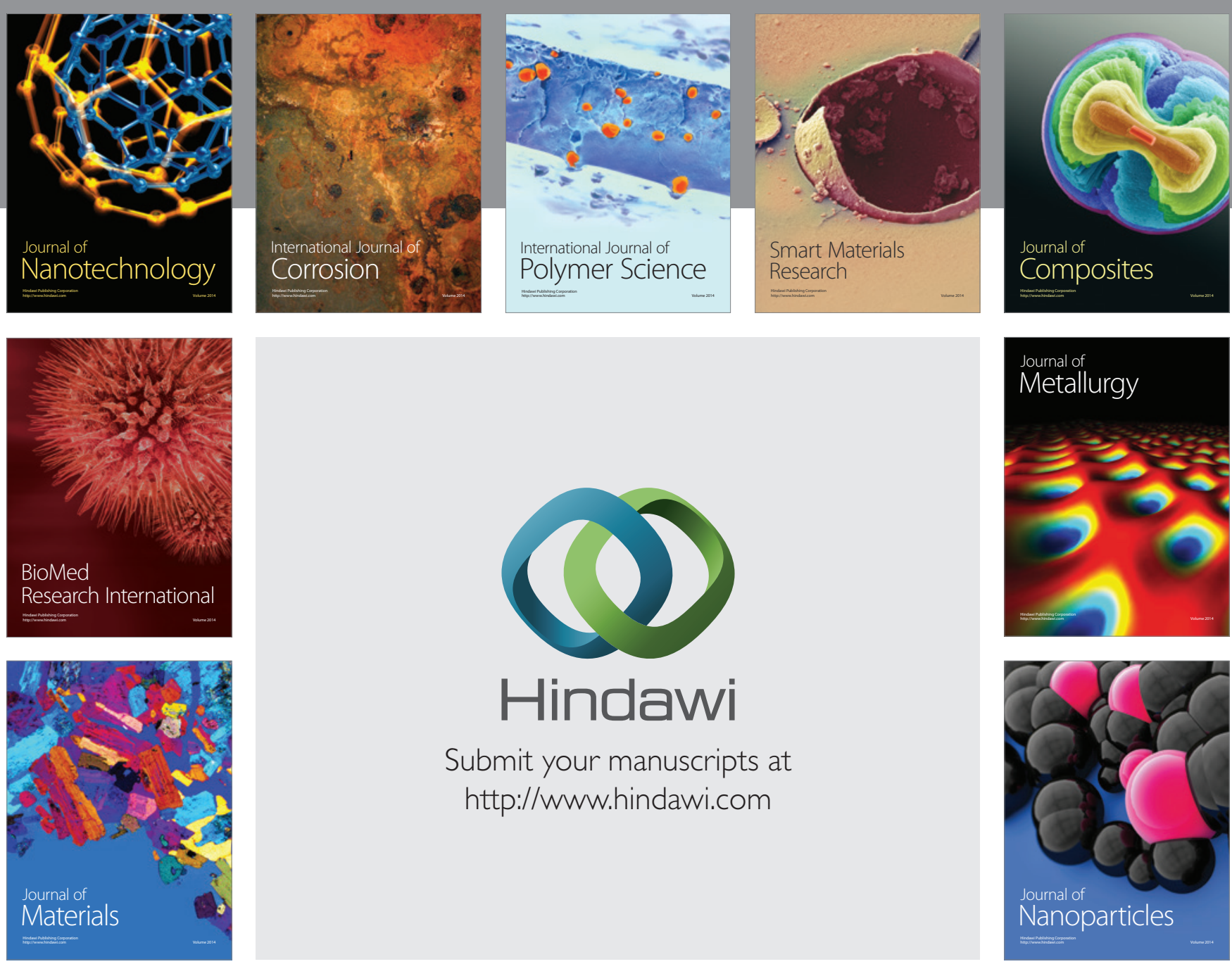

Submit your manuscripts at http://www.hindawi.com
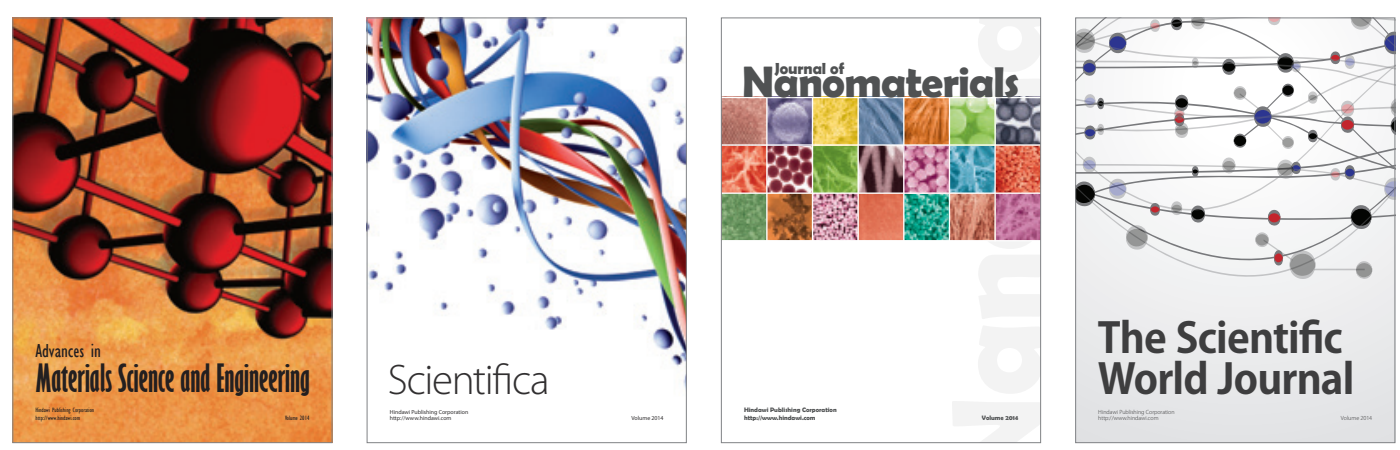

\section{The Scientific World Journal}
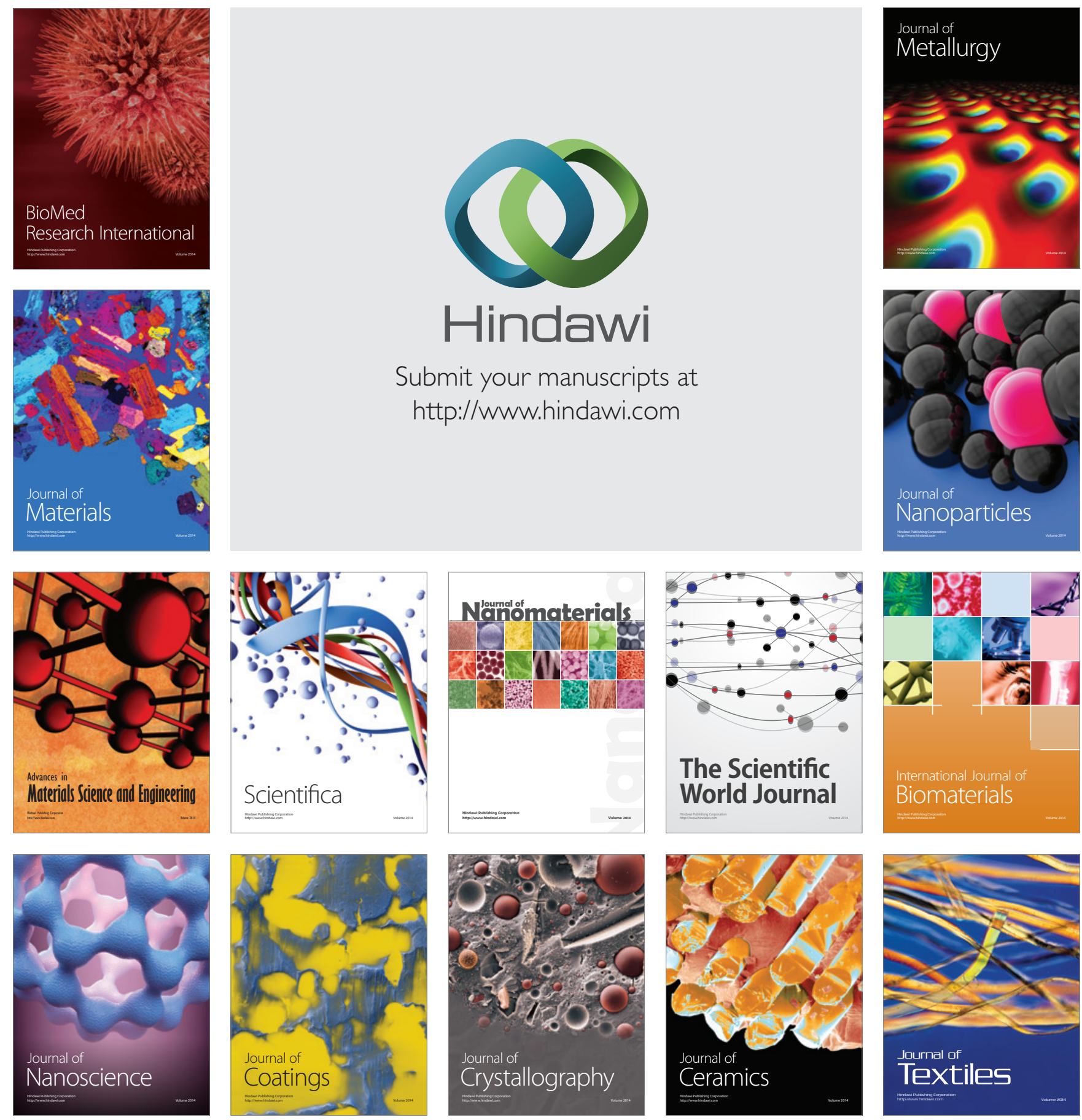